\title{
Synthesis of Macroheterocycles with Ester and Hydrazide Fragments on the Basis of Tetrahydropyran
}

\author{
Gumer Yu. Ishmuratov, ${ }^{@}$ Marina P. Yakovleva, Galina R. Mingaleeva, \\ Rinat R. Muslukhov, Evgeny M. Viripaev, Evgeny G. Galkin, \\ and Alexander G. Tolstikov
}

Institute of Organic Chemistry, Ufa Scientific Centre of RAS, 450054 Ufa, Russia

@Corresponding authorE-mail: insect@anrb.ru

An area of application of tetrahydropyran has been expanded in the directed synthesis of macroheterocycles with ester and hydrazide fragments. The structures of the obtained macrocycles were proved by IR and NMR spectroscopy as well as mass spectrometry. We have already reported the synthesis of tetrahydropyran-based 9-oxo-2E-decenoic acid (2) as the multifunctional pheromone [Chem. Nat. Compd. 2008, 44, 74-76] of queen honeybee Apis melliphera L., 7-oxooctyl-7-oxooctanoate (3) [Bashkir University Bulletin 2008, 3, 466-469 (in Russ.)] and bis(7-oxooctyl)adipate (4) [Butlerov Communications 2009, 17(5), 35-38 (in Russ.)] and also the application of key $\alpha, \omega$-diketones (3, 4) in the directed synthesis of a large variety of macroheterocycles with ester, azine and hydrazide functions, one of which [15,25-dimethyl-1,8-dioxo-16,17,23,24-tetraazacyclohentriaconta-15,24-dien-2,7,18,22-tetraone (10)] exhibited great antibacterial in vitro and in vivo activity [Butlerov Communications 2009, 16(4), 21-25 (in Russ.)]. In this paper we put forward efficient methods to transform tetrahydropyran (1) as a disposable petrochemical product via intermediate $\alpha, \omega$-diketones [7-oxooctyl-7-oxooctanoate (3), bis(7-oxooctyl)adipate (4) and oxabis(ethan-1,2-diyl)(2'E,2'E)bis(9'oxodec-2'-enoate) (5)] into potentially biologically and pharmacologically active macroheterocycles [16,26-dimethyl1,4,7-trioxa-17,18,24,25-tetraazacyclotetratriaconta-9,16,25,32-tetraen-8,19,23,34-tetraone (6), 8,22-dimethyl1-oxa-9,10,20,21-tetraaza-8,21-cyclooctacosadien-2,11,19-trione (8), 8,23-dimethyl-1-oxa-9,10,20,22-teraaza8,22-cyclononakosadien-2,11,20-trione (9), 4,25-dimethyl-28a,29,32,32a-tetrahydro-29,32-epoxy-11,18,2,3,26,27benzadioxatetraazacyclotriaconta-3,25-dien-1,12,17,28-tetraone (11)] that contain the ester and hydrazide functions, including the olefin ones. The macrocycle (6) with conjugated ester groups was synthesized on the basis of unsaturated ketonic acid (2) by its transformation into the appropriate chloroanhydride, [2+1]-condensation with diethylene glycol and subsequent [1+1]-interaction between intermediate diketone diester (5) and glutaric dihydrazide. The synthesis of 28- (8) and 29- (9) member macrolides was performed by [1+1]-condensation of $\alpha, \omega$-dimethylketone (3) with azelaic and sebacic dihydrazides, respectively. The macrolide analog (10) exhibiting the antibacterial activity, i.e., macrocycle (11) with 7-oxabicyclo[2.2.1] heptane fragment in the form of a single di-exo-isomer, was obtained by [1+1]-condensation of the key precursor (4) with the linear dihydrazide of 7-oxabicyclo[2.2.1] hepta-5-en-2,3-dicarbonic acid. The latter, in its turn, was synthesized from dimethyl ester 7-oxabicyclo[2.2.1] hept-5-en-2,3-dicarbocylic acid mixed with the cyclic derivative [2,3,4a,5,8,8a-hexahydro-5,8-epoxyphtalazin-1,4-dione] and separated from the reaction mixture. In our opinion, the introduction of pharmacophoric 7-oxabicyclo[2.2.1] heptane fragment [J. Med. Chem. 1985, 28, 15801590; Heterocycles 1978, 9, 1749-1757] will serve to increase the known pharmacological activity and display its new types. It should be noted that all macrocyclization reactions were carried out at room temperature in dioxane under high-dilution conditions. The structures of the obtained macrocycles $(\mathbf{6}, \mathbf{8}, \mathbf{9}, \mathbf{1 1})$ were determined by IR, NMR ${ }^{1} H$ and ${ }^{13}$ C spectroscopy; their purity was HPLC controlled.

Keywords: Tetrahydropyran, $\mathrm{O}, \mathrm{N}$-macroheterocycles, ester and hydrazide functions, synthesis. 


\title{
Синтез макрогетероциклических соединений, содержащих эфирные и гидразидные фрагменты, из тетрагидропирана
}

\author{
Г. Ю. Ишмуратов, М. П. Яковлева, Г. Р. Мингалеева, Р. Р. Муслухов, \\ Е. М. Вырыпаев, Е. Г. Галкин, А. Г. Толстиков
}

Учреждение Российской академии наук Институт органической химии Уфимского научного иентра Российской академии наук, 450054 г. Уфа, Россия

E-mail:insect@anrb.ru

\begin{abstract}
Расширена область применения тетрагидропирана в направленном синтезе макрогетероциклов с эфирныли и гидразидными функциями, в том числе олефиновых. Приведено доказательство строения полученных макрочиклов методами ИК- и ЯМР-спектроскопии и масс-спектрометрии.
\end{abstract}

Ключевые слова: Тетрагидропиран, макрогетероциклы, простые и сложные эфиры, гидразиды, синтез.

\section{Введение}

Ранее нами сообщалось о синтезе из тетрагидропирана (1) многофункционального феромона (2 $)^{[1]}$ матки медоносной пчелы Apis melliphera L., дикетоэфира (3) и дикетодиэфира (4) ${ }^{[4]}$ и использовании ключевых $\alpha, \omega$ дикетонов $(3,4)$ в направленном синтезе целого ряда макрогетероциклов со сложноэфирными, азинной и гидразидными функциями, один из которых, 15,25-диметил1,8 -диоксо-16,17,23,24-тетраазациклогентриаконта15,24-диен-2,7,18,22-тетраон, проявил значительную антибактериальную in vitro и in vivo активность. ${ }^{[4]}$

В данном сообщении нами предложены эффективные пути превращения доступного нефтехимического продукта - тетрагидропирана (1) - через промежуточные $\alpha, \omega$-дикетоны (3-5) в потенциально биологически и фармакологически активные макрогетероциклы $(\mathbf{6}, \mathbf{8}, 9, \mathbf{1 1})$ с эфирными и гидразидными функциями, в том числе и олефиновые.

\section{Результаты и обсуждение}

Макроцикл (6) с сопряженными сложноэфирными группами синтезирован исходя из ненасыщенной кетокислоты (2) превращением в соответствующий хлорангидрид,

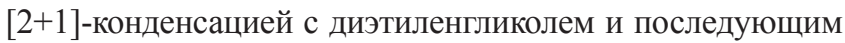
$[1+1]$-взаимодействием промежуточного дикетодиэфира (5) с дигидразидом глутаровой кислоты (12).

Синтез 28- (8) и 29- (9) -членных макролидов выполнен $[1+1]$-конденсацией $\alpha, \omega$-диметилкетона (3) с дигидразидами азелаиновой (14) и себациновой (15) кислот соответственно.

Аналог проявившего антибактериальную активность макролида 10 $\mathbf{1 0}^{[4]}$ - макроцикл 11 с 7-оксабицикло [2.2.1] гептеновым фрагментом в виде единственного ди-экзо-изомера - получен $[1+1]$-конденсацией ключевого предшественника (4) с линейным дигидразидом 7-оксабицикло[2.2.1]гепт-5-ен-2,3-дикарбоновой кислоты (16). Последний, в свою очередь, синтезирован из диметилового эфира (17) в смеси с циклическим производным 18 и выделен из неё. Введение фармакофорного 7-оксабицикло[2.2.1]гептенового фрагмента, ${ }^{[5-7]}$ по нашему мнению, будет способствовать усилению известной и проявлению новых видов фармакологической активности.

Отмечаем, что все реакции макроциклизации выполнены при комнатной температуре в диоксане в условиях высокого разбавления (Схема 1).

Структуры полученных макроциклов 6, 8, 9, 11 установлены методами ИК, ${ }^{1} \mathrm{H}$ и ${ }^{13} \mathrm{C}$ ЯМР спектроскопии, чистота контролировалась ВЭЖХ и составляла не менее 95\%. Анализ спектров ЯМР проводили сравнением со спектрами исходных соединений: дикетоэфиров (3-5) и гидразидов соответствующих дикарбоновых кислот (12, 14-16).

Так, в спектре ${ }^{13} \mathrm{C}$ ЯМР продукта реакции 6 отсутствуют сигналы исходного дикетоэфира 5: карбонильных атомов углерода (208.75 м.д.) и соответствующих атомам углерода $\mathrm{CH}_{2}$ - и $\mathrm{CH}_{3}$-групп в $\alpha$-положении к кетогруппам (43.26 и 29.69 м.д.). Кроме сигнала атомов углерода сложноэфирных групп (175.56 м.д.) и смещенного по сравнению с исходным дигидразидом (171.89 м.д.) сигнала атомов углерода групп NH-C=O (175.56 м.д.), присутствует синглетный сигнал групп $\mathrm{C}=\mathrm{N}$ (151.78 м.д.). Кроме того, в протонном спектре макроцикла 6 нет сигнала (4.75 м.д.) гидразидного $\left(\mathrm{NH}_{2} \mathrm{NH}\right)$ остатка, а имеется слабопольный сигнал (8.50 м.д.), величина химического сдвига и интегральная интенсивность которого соответствует двум амидным протонам $\mathrm{NHC}=\mathrm{O}$ групп макроцикла. Эти факты указывают на то, что полученное соединение не является ациклическим продуктом замещения и содержит в своем составе гидразидные фрагменты.

В углеродном спектре макроцикла 11, записанном в $\mathrm{CDCl}_{3}$, наблюдаются двойные сигналы атомов фуранового цикла в гидразидном фрагменте: имеются соответственно по два дублетных сигнала атомов С-29, C-32 (80.46 и 78.64 м.д.) и С-28a, C-32а (48.68 и 45.87 


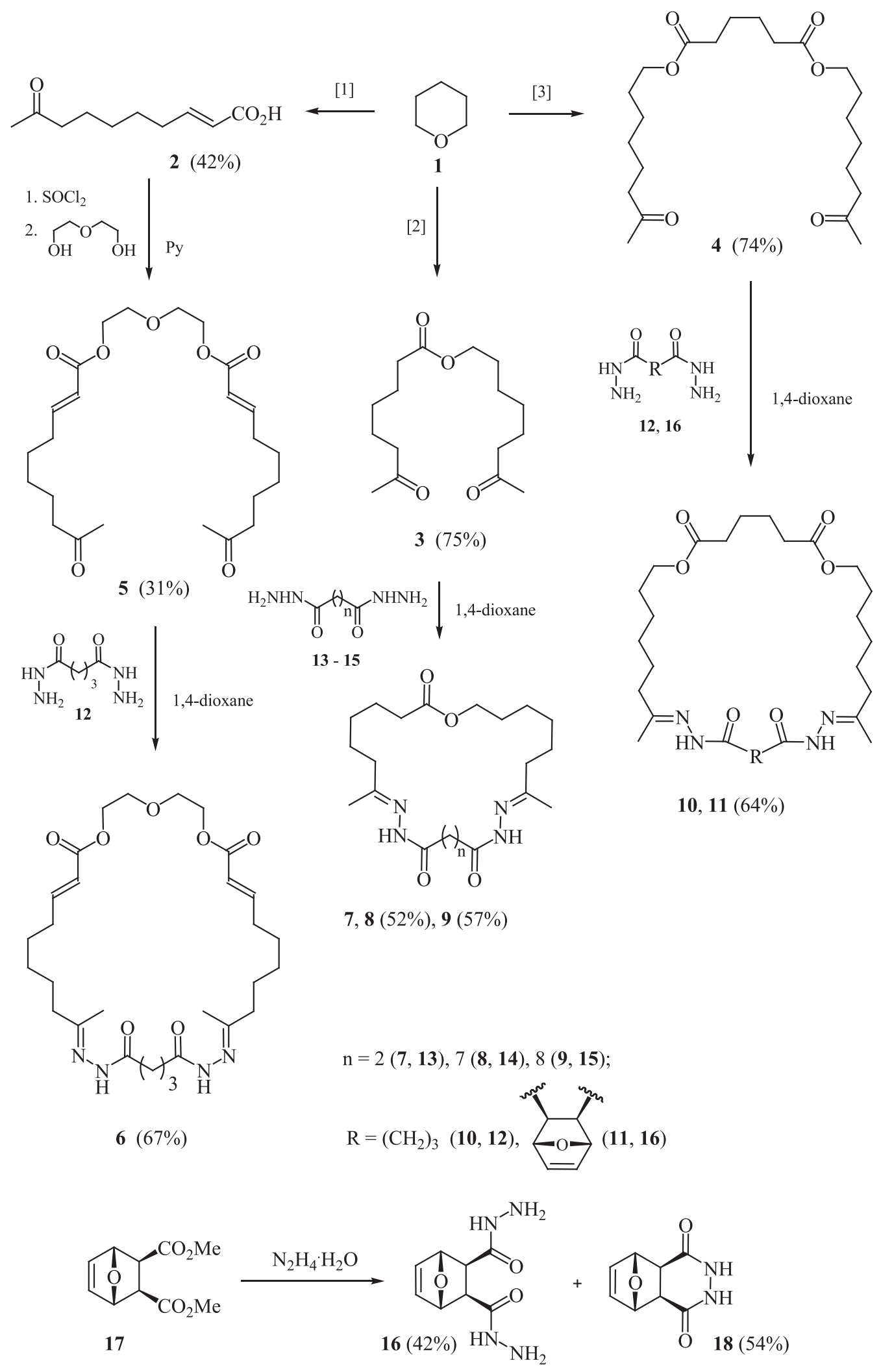

Схема 1.

м.д.), а также атомов углерода амидных (C(O)NH) групп (171.08 и 171.83 м.д.) с соотношением интенсивностей $2: 1$. О преимущественном образовании транс-трансстереоизомера также свидетельствует преобладание первого из двух (16.32 и 22.52 м.д.) квартетных сигналов $\mathrm{CH}_{3}$-групп. В спектре ${ }^{13} \mathrm{C}$ ЯМР макролида 11 , регистрированном в $\mathrm{DMSO}-\mathrm{d}_{6}\left(50^{\circ} \mathrm{C}\right)$, соотношение интенсивностей значительно изменяется. Из этого можно пред- положить, что макрогетероцикл 11 представляет смесь двух конформеров, устойчивость которых, вероятно, определяется как стерическими факторами, так и амидным сопряжением в гидразидном фрагменте молекулы.

Молекулярные массы макролидов 6, 8, 9, 11 устанавливались методами масс-спектрометрии. Поскольку исследуемые соединения представляют собой весьма нестойкие аддукты, особенно в условиях применяемого 
при масс-спектрометрировании глубокого вакуума, то первоначально были получены спектры химической ионизации при атмосферном давлении (ХИАД, АРСІ, 20 эВ) с регистрацией положительных и отрицательных ионов на приборе LC MS 2010 фирмы «Shimadzu», содержащем соединение жидкостного хроматографа (жидкая подвижная фаза - вода, ацетонитрил) с массспектрометрическим детектором. При этом были зарегистрированы весьма интенсивные пики протонированных $\mathrm{MH}^{+}$и депротонированных (M-H)- ионов, а также их

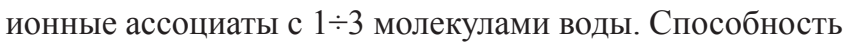
к протонированию и сольватизации с водой хорошо известные факты в химии амидов и гидразидов. ${ }^{[8]}$ Этим было обусловлено первоначальное обращение к методике ХИАД.

Для подтверждения строения продуктов 8, 9 были получены их масс-спектры с ионизацией электронным ударом (ЭУ) и проведено определение точных массовых чисел большинства значимых пиков ионов. Поскольку они содержат сложноэфирные и две гидразидные группы, разделенные друг от друга соответственно 7 и 8 метиленовыми звеньями, то для сравнения были использованы полученные с высоким разрешением массспектры исходного дикетоэфира 3, ранее полученного макролида $7^{[9]}$ и дигидразидов 13-15, как из каталога, так и синтезированных образцов. Предполагалось, что основные фрагменты модельных спектров будут наблюдаться и при распаде МИ исследуемых соединений и существенно облегчат их интерпретацию.

В масс-спектрах ЭУ соединений 7-9 зарегистрированы пики молекулярных ионов (МИ, $\left.\mathrm{M}^{+}\right)$, точное измерение массовых чисел которых дало хорошее соответствие предполагаемым брутто-составам. Интенсивность пиков МИ очень мала в спектре (7) - $1.3 \%$ и значительна в спектрах 8, 9 - 53.2 и $64.4 \%$, соответственно. Это в какой-то степени согласуется с интенсивностью пиков МИ исходных дигидразидов (13-15 - 0, 7.5 и 7.0\% от максимального пика, соответственно). Альтернативное объяснение: МИ соединения 7 существует исключительно в раскрытой форме, а для $\mathbf{8 , 9}$ предпочтительна циклическая структура, хотя не исключается и наличие раскрытых форм.

Раскрытию цикла благоприятствует наличие функциональных групп, способных к внутримолекулярному протонированию (карбонильных, амидных, иминных), причем миграции атомов водорода могут осуществляться как по традиционным механизмам через 4-х и 6-членные (перегруппировка типа Мак-Лафферти) переходные состояния, так и вследствие наличия достаточно длинных и конформационно гибких полиметиленовых цепочек путем неспецифических миграций атомов водорода при пространственном сближении с потенциальными акцепторами протонов. ${ }^{[10]}$ Принято считать, ${ }^{[11,12]}$ что это атомы водорода, находящиеся преимущественно в $\alpha$-положении к другим функциональным группам. С этими процессами могут конкурировать процессы кетоенольной таутомерии, которые могут и стабилизировать пик МИ (появление сопряженных систем двойных связей в 8,9 ), так и способствовать реализации новых путей распада.

Прежде всего, аналитически значимыми представляются фрагменты, соответствующие потере алкилдигидразидного и гидразидного осколков (Схема 2). Первые регистрировались во всех масс-спектрах соединений 7-9, а вторые лишь в 8, 9. Осколочным ионам на Схеме 1 по аналогии с ранее изученными краун-эфирами ${ }^{[11,12]}$ приписаны структуры протонированных МИ более низкомолекулярных гомологов.

Комплементарные ионы состава $\left[\mathrm{C}_{4} \mathrm{H}_{6} \mathrm{~N}_{2} \mathrm{O}_{2}\right]$ с $m / z 114.0439$ (рассчитано: 114.0424) и $\left[\mathrm{C}_{3} \mathrm{H}_{6} \mathrm{~N}_{2} \mathrm{O}\right] \mathrm{c} \mathrm{m} / \mathrm{z}$ 86.0497 (рассчитано: 86.0475), наблюдавшиеся в 7, регистрировались в спектре исходного дигидразида 13 как пики ионов $\left[\mathrm{M}-\mathrm{NH}_{2} \mathrm{NH}_{2}\right]^{+}$и $\left[\mathrm{M}-\mathrm{NH}_{2} \mathrm{NH}_{2} \mathrm{CO}\right]^{+}$при, соответственно, $\mathrm{m} / \mathrm{z} 114.0389$ и 86.0464. Фрагмент с $\mathrm{m} / \mathrm{z}$ 114 в спектре 8, согласно точно измеренному значению (114.0858), соответствовал составу $\left[\mathrm{C}_{5} \mathrm{H}_{10} \mathrm{~N}_{2} \mathrm{O}\right]$ (рассчитано: 114.0793).

Информативные пики ионов $\left[\mathrm{M}-\mathrm{C}_{11} \mathrm{H}_{19} \mathrm{O}_{2}\right]^{+}$наблюдались в масс-спектрах соединений 8,9 (Схема 3) и соответствовали потере части фрагмента со сложноэфирной группой.

В масс-спектрах 7-9 также регистрировались фрагменты с $m / z$ 141.0940, 141.0939, 141.0924, которые соответствовали составу $\left[\mathrm{C}_{8} \mathrm{H}_{13} \mathrm{O}_{2}\right]$ (рассчитано: 141.0910). Ион такого состава был одним из основных в спектре модельного дикетоэфира 3 и, очевидно, имел структуру $\left[\mathrm{CH}_{3}-\mathrm{C}(=\mathrm{O}) \mathrm{C}_{5} \mathrm{H}_{10}-\mathrm{C} \equiv \mathrm{O}^{+}\right]$. Но в исследуемых соединениях он образован другими атомами макроцикла и, предположительно, имеет структуры $\left[\mathrm{CH}_{2}=\mathrm{CH}-\mathrm{C}(=\mathrm{O})-\mathrm{OC}_{4} \mathrm{H}_{8}^{+}\right]$ или $\left[{ }^{+} \mathrm{CH}_{2}-\mathrm{CH}=\mathrm{CH}(\mathrm{OH})-\mathrm{OC}_{3} \mathrm{H}_{6} \mathrm{CH}=\mathrm{CH}_{2}\right]$. Искомый же

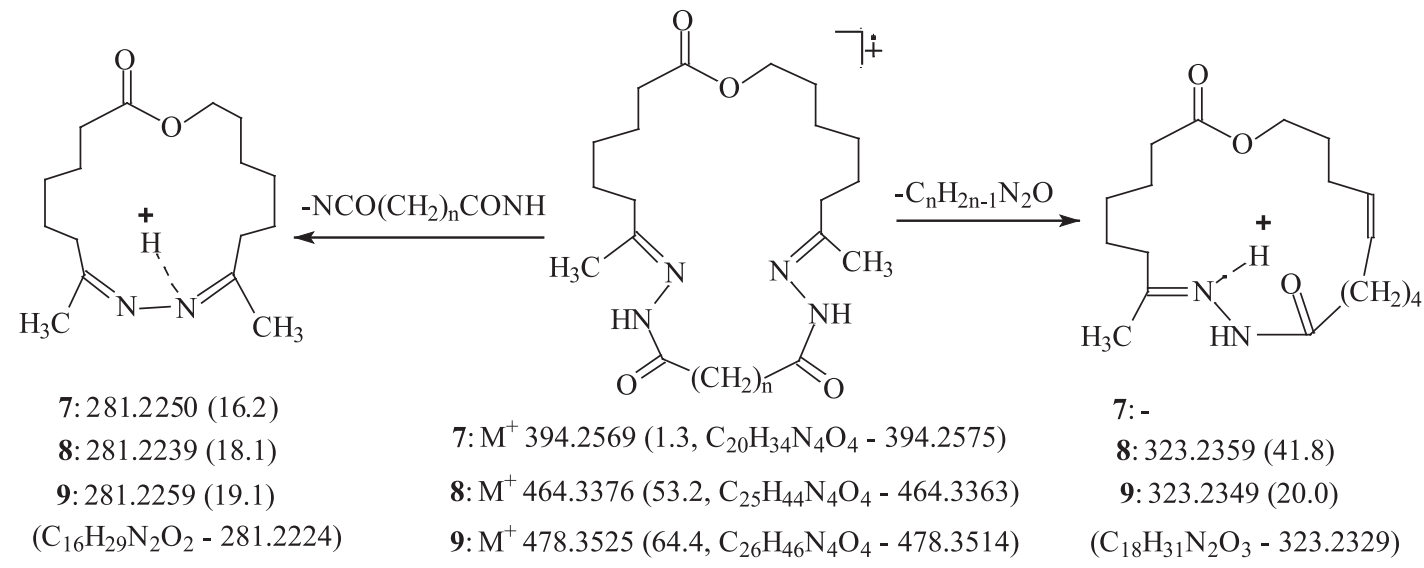

Схема 2. 
аналог модельного фрагмента обнаруживается в спектрах 8, 9 при $m / z 155.1197$ и имеет состав $\left[\mathrm{C}_{8} \mathrm{H}_{15} \mathrm{~N}_{2} \mathrm{O}\right]$ (рассчитано: 155.1184), т.е. он содержит вместо ацетильного кислорода HNNH- группу.

Для фрагментации МИ соединения 7 характерны разрывы связей $\mathrm{N}-\mathrm{C}(\mathrm{O})$ и $\mathrm{C}-\mathrm{C}$ в $\beta$-положении к двойным связям. В качестве одного из основных образовывался фрагмент $m / z 154.0733$ состава $\left[\mathrm{C}_{7} \mathrm{H}_{10} \mathrm{~N}_{2} \mathrm{O}_{2}\right.$ ] (рассчитано: 154.0737). Он получался либо путем двух простых разрывов (1), либо путем раскрытия цикла и последующего отрыва за счет двух последовательных перегруппировок Мак-Лафферти (2) $)^{[11]}$ (Схема 4).

В масс-спектре ИЭ 7 зарегистрированы также фрагменты $[\mathrm{M}-71]^{+},[\mathrm{M}-137]^{+},[\mathrm{M}-171]^{+}, \quad[\mathrm{M}-227]^{+}$, соответствующие потерям частиц $\mathrm{C}_{3} \mathrm{H}_{7} \mathrm{~N}_{2}, \mathrm{C}_{7} \mathrm{H}_{9} \mathrm{~N}_{2} \mathrm{O}$, $\mathrm{C}_{8} \mathrm{H}_{15} \mathrm{~N}_{2} \mathrm{O}_{2}, \mathrm{C}_{12} \mathrm{H}_{23} \mathrm{~N}_{2} \mathrm{O}_{2}$. Пики ионов [M-71] $]^{+}$, вероятно, получались за счет сочетания перегруппировки МакЛафферти и разрыва у карбонильного атома углерода (Схема 5), соответствовали отрывам $\mathrm{C}_{3} \mathrm{H}_{7} \mathrm{~N}_{2}$ и наблюдались также и в масс-спектрах $\mathbf{8 , 9}$ при соответственно $\mathrm{m} / \mathrm{z}$ $393.2791\left[\mathrm{C}_{22} \mathrm{H}_{37} \mathrm{~N}_{2} \mathrm{O}_{4}\right]$ (рассчитано: 393.2753 ) и 407.2887 $\left[\mathrm{C}_{23} \mathrm{H}_{39} \mathrm{~N}_{2} \mathrm{O}_{4}\right]$ (рассчитано: 407.2904). Эти фрагменты, в отличие от 7, в спектрах 8,9 далее теряли $\mathrm{CO}, \mathrm{COCH}_{2}$, $\mathrm{COC}_{4} \mathrm{H}_{8}$ и т.д. Комплементарный пик иона состава [ $\left.\mathrm{C}_{3} \mathrm{H}_{8} \mathrm{~N}_{2}\right]$ (измерено: 72.0665, рассчитано: 72.0682) был основным в спектрах 7,8 и одним из основных в спектре 9.

В масс-спектрах соединений 8,9 наблюдается множество интенсивных линий, между тем как для исходных соединений $3,14,15$ число их хотя и велико, но они очень малы (интенсивность не превышает 30\% от максимального пика).

Масс-спектры 8 и 9 довольно близки по интенсивности пиков МИ, наличию ряда фрагментов, полученных за счет отрыва одинаковых частиц. Рост числа метиленовых звеньев между гидразидными группами приводит к появлению не наблюдавшихся в спектре 7 интенсивных фрагментов $\left[\mathrm{M}-\mathrm{CH}_{3}\right]^{+},\left[\mathrm{M}-\mathrm{C}_{3} \mathrm{H}_{7}\right]^{+},\left[\mathrm{C}_{2} \mathrm{H}_{4} \mathrm{NO}\right]^{+}$в спектрах $\mathbf{8}, \mathbf{9}$. Аналитически значимой представляется группа ионов высокой распространенности состава $\left[\mathrm{C}_{13} \mathrm{H}_{24} \mathrm{~N}_{2} \mathrm{O}_{3}\right]$, $\mathrm{m} / z 256.1798$ (94.1\%) (рассчитано: 256.1787) для 8; $\left[\mathrm{C}_{14} \mathrm{H}_{26} \mathrm{~N}_{2} \mathrm{O}_{3}\right], m / z 270.1936$ (62.6\%) (рассчитано: 270.1938 ) для 9; $\left[\mathrm{C}_{12} \mathrm{H}_{21} \mathrm{~N}_{2} \mathrm{O}_{3}\right], m / z 241.1561$ (87.2\%) (рассчитано: 241.1552) для 8, $\left[\mathrm{C}_{13} \mathrm{H}_{23} \mathrm{~N}_{2} \mathrm{O}_{3}\right], m / z \quad 255.1695 \quad(77.7 \%)$ (рассчитано: 255.1703) для 9; $\left[\mathrm{C}_{11} \mathrm{H}_{17} \mathrm{~N}_{2} \mathrm{O}_{2}\right], \mathrm{m} / z$ 209.1295 (51.1\%) (рассчитано: 209.1290) для 8, [C $\left.{ }_{12} \mathrm{H}_{19} \mathrm{~N}_{2} \mathrm{O}_{2}\right], \mathrm{m} / \mathrm{z}$ 223.1436 (59.8\%) (рассчитано: 223.1441) для 9; которые, располагаясь в средней массовой области спектров и характеризуясь близкими интенсивностями, различаются в 8 и 9 на величину $m / z$, равную гомологической разности. Это свидетельствует о том, что в их состав полностью или частично входят полиметиленовые цепи, расположенные между дигидразидными группами.

Итак, в результате масс-спектрометрического исследования макролидов 7-9 можно сделать следующие выводы:

1) поскольку в спектрах ХИАД были зарегистрированы пики протонированных положительных $\mathrm{MH}^{+}$и депротонированных отрицательных (М-H)- ионов для всего ряда продуктов 7-9, то проявление этой тенденции

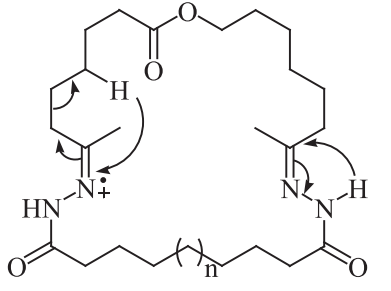

8: $\mathrm{C}_{24} \mathrm{H}_{44} \mathrm{~N}_{4} \mathrm{O}_{4} 464.3376$ 9: $\mathrm{C}_{25} \mathrm{H}_{46} \mathrm{~N}_{4} \mathrm{O}_{4} 478.3549$<smiles>C=CCCC(=O)OCCCCCCC(C)N=NC(=O)CCCCCCCCC(=O)NNC(=C)C</smiles><smiles>CCCN=C(C)CC(C)N=NC(=O)CCCCCCCC(=O)N=N</smiles>

$\mathrm{C}_{14} \mathrm{H}_{25} \mathrm{~N}_{4} \mathrm{O}_{2} 281.1994$ (281.1972) $\mathrm{C}_{15} \mathrm{H}_{27} \mathrm{~N}_{4} \mathrm{O}_{2} 295.2090$ (295.2129)

\section{Схема 3.}

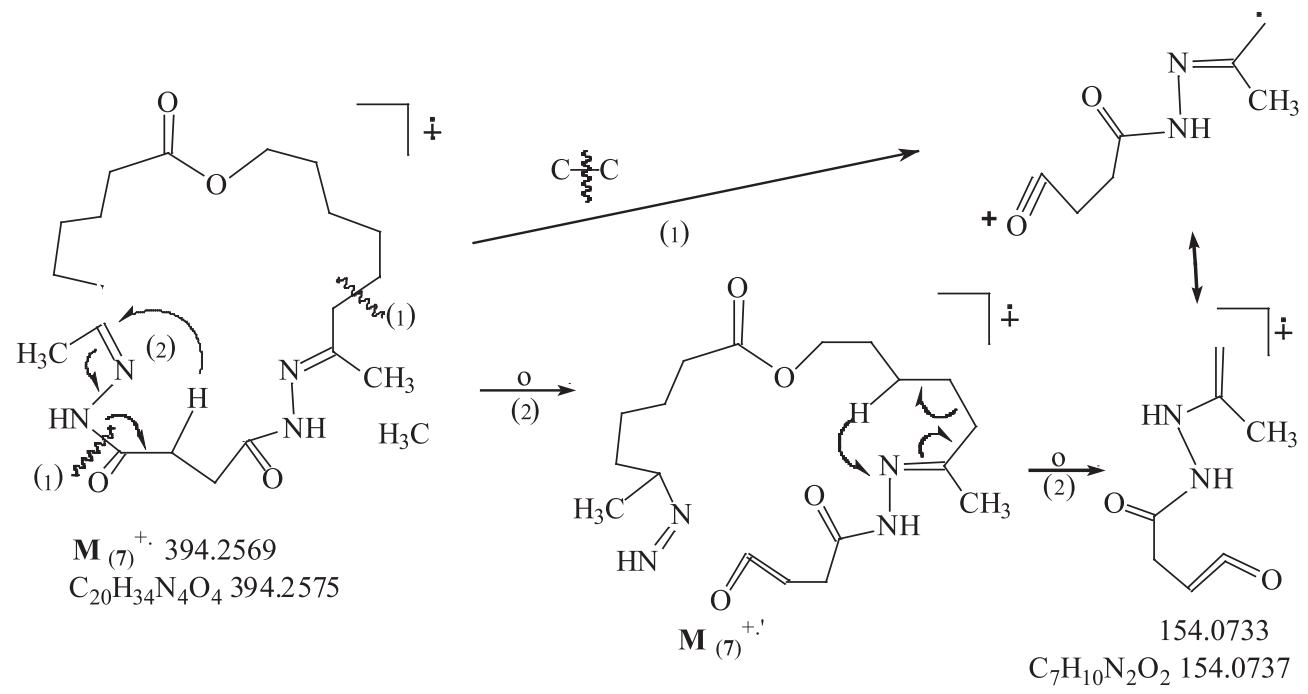

Схема 4. 

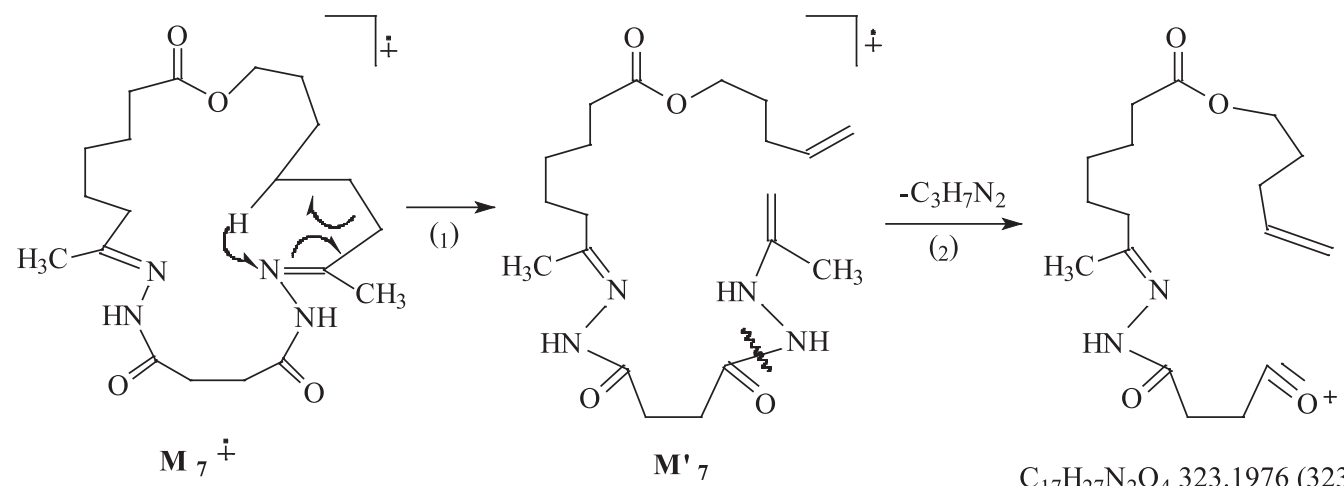

Схема 5.

можно считать за доказательство существования соединений с соответствующими молекулярными массами;

2) анализ структурно-специфических ионов, проведенный согласно известным литературным данным, ${ }^{[10-12]}$ позволяет интерпретировать масс-спектры с ионизацией электронами (7-9) как спектры соответственно 23-, 28- и 29-членных макролидов с гидразидными фрагментами;

3) стабильность пиков ионов $\mathrm{M}^{+}$и особенности их фрагментации существенно зависят от длины полиметиленовой цепочки между гидразидными группами: с её увеличением интенсивность пиков $\mathrm{M}^{+}$возрастает и наблюдаются новые каналы рапада.

\section{Выводы}

Расширена область применения доступного нефтехимического продукта - тетрагидропирана - в направленном синтезе потенциально биологически и фармакологически активных макрогетероциклов 6-9, 11 с эфирными и гидразидными функциями, в том числе и олефиновых, с использованием [1+1]-конденсации промежуточных $\alpha, \omega$-дикетодиэфиров с соответствующими гидразидами дикарбоновых кислот. Строение полученных макроциклов установлено методами ИК- и ЯМР-спектроскопии и масс-спектрометрии, чистота контролировалась высокоэффективной жидкостной хроматографией.

\section{Экспериментальная часть}

ИК-спектры записывали на приборе UR-20 в тонком слое. Спектры ЯMP регистрировали на спектрометре «BRUKER AM-300» (рабочая частота 300.13 МГц для ${ }^{1} \mathrm{H}$ и 75.47 МГц для ${ }^{13} \mathrm{C}$ ) в растворах $\mathrm{CDCl}_{3}$ и DMSO-d $\mathrm{d}_{6}$ с внутренним стандартом TMS и $\mathrm{D}_{2} \mathrm{O}$ с внутренним стандартом DSS. Анализы BЭЖX выполнены на жидкостном хроматографе «Shimadzu - LC20AD» с диодноматричным детектором SPD-M20A (Shimadzu, Япония) с использованием колонки Phenomenex 250 х 4.6 мм (сорбент - Luna C18 с диаметром частиц 5 мкм). В качестве подвижной фазы применялся элюент (вода - ацетонитрил) со скоростью потока 1 мл/мин. Аналитическая длина волны 215 нм. Для колоночной хроматографии использовали $\mathrm{SiO}_{2}$ (70230) марки «Lancaster» (Англия). Контроль ТCX осуществляли на $\mathrm{SiO}_{2}$ марки Sorbfil (Россия). Данные элементного анализа всех соединений отвечали вычисленным. Масс-спектры соединений сняты на приборе LC MS 2010 «Shimadzu» в условиях ХИАД при энергии электронов 20 эВ с регистрацией положительных и отрицательных ионов. Жидкая подвижная фаза вода и(или) ацетонитрил при скоростях потока носителя 0.02 мл/мин. Масс-спектры электронного удара получены на хромато-масс-спектрометре Finnigan MAT 95 XL с системой обработки данных DEC Alpha Station 433 аи при температуре источника ионов $250^{\circ} \mathrm{C}$ и энергии ионизирующих электронов 70 эВ, прямой ввод проб. Точное определение массовых чисел ионов проведено в диапазоне 1-1000 D при разрешении 10000 на высоте $10 \%$, стандарт - перфторкеросин.

Оксабис(этан-1,2-диил)(2 'E, 2' Е)бис(9'-оксодеи-2'еноат) (5). Смесь 9-оксо-2Е-деценовой кислоты (2) (1.00 г, 5.43 ммоль) и $\mathrm{SOCl}_{2}\left(1.29\right.$ г, 10.86 ммоль) нагревали при $60^{\circ} \mathrm{C}$ до окончания выделения газов, затем отгоняли избыток $\mathrm{SOCl}_{2}$ при пониженном давлении. К перемешиваемому остатку хлорангидрида 9-оксо-2Е-деценовой кислоты [ИК $(\mathrm{KBr}) v_{\max }$ $\mathrm{cm}^{-1}$ : $\left.1753(\mathrm{COO}), 1719(\mathrm{C}=\mathrm{O}), 1626(\mathrm{C}=\mathrm{C}), 486(\mathrm{C}-\mathrm{Cl})\right]$ в 0.5 мл абсолютного $\mathrm{Et}_{2} \mathrm{O}$ прибавляли по каплям раствор абсолютного диэтиленгликоля (0.18 г, 1.71 ммоль) в 0.5 мл абсолютного пиридина. Реакционную смесь выдерживали при комнатной температуре 6 ч (контроль ТСХ), затем прибавляли 4 мл $\mathrm{CH}_{2} \mathrm{Cl}_{2}$, последовательно промывали $5 \% \mathrm{HCl}$, холодными насыщенными растворами $\mathrm{NaHCO}_{3}$ и $\mathrm{NaCl}$, сушили $\mathrm{MgSO}_{4}$, упаривали, остаток хроматографировали $\left(\mathrm{SiO}_{2}\right.$, ПЭ-МТБЭ, 1:1). Выход 0.22 г (31\%). Масс-спектр (АРCI, 20 эВ), $m / z$ : 437 [M-H], 455 $\left[\mathrm{M}-\mathrm{H}^{+} \mathrm{H}_{2} \mathrm{O}\right]^{-}, 473\left[\mathrm{M}-\mathrm{H}+2 \mathrm{H}_{2} \mathrm{O}\right]^{-}$. ИК $(\mathrm{KBr}) v_{\max } \mathrm{cm}^{-1}: 1718(\mathrm{COO})$, $1701(\mathrm{C}=\mathrm{O}), 1653(\mathrm{C}=\mathrm{C}) .{ }^{1} \mathrm{H}$ ЯМР $\left(\mathrm{CDCl}_{3}\right) \delta$ м.д.: 1.37-1.42 (4H, м, Н-5'), 1.45-1.57 (8H, м, Н-6', Н-7'), 2.10 (6H, с, H-10'), 2.102.23 (4Н, м, Н-4'), 2.41 (4Н, т $J=7.3$ Гц, Н-8'), 3.28-3.64 (4Н, м, Н-2), 4.26 (4Н, т $J=6.8$ Гц, Н-1), 5.82 (2Н, д $J=15.6$ Гц, Н-2'), 6.93 $\left(2 \mathrm{H}\right.$, дт $J=15.6$ Гц, $J=7.0$ Гц, Н-3'). ${ }^{13} \mathrm{C}$ ЯМР $\left(\mathrm{CDCl}_{3}\right) \delta$ м.д.: 23.22 (т, C-7'), 27.50 (т, С-6'), 28.37 (т, C-5'), 29.69 (к, С-10'), 31.76 (т, С-4'), 43.26 (т, С-8'), 63.06 (т, С-1), 68.92 (т, С-2), 120.78 (д, С-2'), 149.48 (д, С-3'), 166.31 (с, С-1'), 208.75 (с, С-9').

16,26-Диметил-1,4,7-триокса-17,18,24,25тетраазаииклотетратриаконта-9, 16,25,32-тетраен8,19,23,34-тетраон (6). К интенсивно перемешиваемому раствору дикетодиэфира 5 (0.10 г, 0.22 ммоль) в 1.9 мл абсолютного диоксана медленно прибавляли дигидразид глутаровой кислоты (0.03 г, 0.22 ммоль), полученный согласно ${ }^{[5]}$. Выдерживали в течение 48 ч (контроль ТСХ), диоксан упаривали. Остаток растворяли в 5 мл $\mathrm{CH}_{2} \mathrm{Cl}_{2}$, промывали водой $(3 \times 1$ мл), сушили $\mathrm{MgSO}_{4}$ и упаривали. К полученному остатку при перемешивании добавляли последовательно 0.5 мл абсолютного $\mathrm{CH}_{2} \mathrm{Cl}_{2}$ и 5 мл гексана, выдерживали до разделения слоев, верхний из которых декантировали. Остаток промывали 2 мл гексана и упаривали. Выход 0.08 г (67\%). Масс-спектр (АРСІ, 20 эB), $m / z: 561[\mathrm{M}-\mathrm{H}]^{-}, 579\left[\mathrm{M}-\mathrm{H}^{+} \mathrm{H}_{2} \mathrm{O}\right]^{-}, 597\left[\mathrm{M}-\mathrm{H}+2 \mathrm{H}_{2} \mathrm{O}\right], 615$ $\left[\mathrm{M}-\mathrm{H}+3 \mathrm{H}_{2} \mathrm{O}\right]^{-}, 563[\mathrm{M}+\mathrm{H}]^{+}, 581\left[\mathrm{M}+\mathrm{H}+\mathrm{H}_{2} \mathrm{O}\right]^{+}$. ИК $(\mathrm{KBr}) v_{\max }$ $\mathrm{cm}^{-1}: 3197(\mathrm{NH}), 1718(\mathrm{COO}), 1699(\mathrm{CONH}), 1647(\mathrm{C}=\mathrm{C}), 1627$ $(\mathrm{C}=\mathrm{N}) .{ }^{1} \mathrm{H}$ ЯМР $\left(\mathrm{CDCl}_{3}\right) \delta$ м.Д.: 1.27-1.40 (2H, м, H-21), 1.40-1.62 (4H, м, H-12, H-30), 1.48-1.62 (8H, м, H-13, H-14, H-28, H-29), 1.77 (6H, c, $\left.\mathrm{CH}_{3}-16, \mathrm{CH}_{3}-26\right), 2.18-2.24$ (4H, м, H-11, H-31), 2.37 
(4H, т $J=7.3$ Гц, Н-20, Н-22), 2.71 (4Н, т $J=7.1$ Гц, Н-15, H-27), 3.65-3.80 (4H, м, H-3, H-5), 4.20-4.39 (4H, м, H-2, H-6), 5.58 (2H, д $J=15.7$ Гц, Н-9, Н-33), 6.59 (2Н, дт $J=15.7$ Гц, $J=6.9$ Гц, Н-10, $\mathrm{H}-32), 8.50$ (2H, уш.с, NH). ${ }^{13} \mathrm{C}$ ЯMP $\left(\mathrm{CDCl}_{3}\right) \delta$ м.д.: 15.96 (к, $\left.\mathrm{CH}_{3}-16, \mathrm{CH}_{3}-26\right), 19.52$ (т, C-21), 25.92 (т, C-14, C-28), 27.76 (т, C-13, C-29), 28.63 (т, C-12, C-30), 32.04 (т, C-11, C-31), 37.79 (т, C-20, C-22), 38.66 (т, C-15, C-27), 63.38 (т, C-2, С-6), 69.15 (т, С-3, C-5), 121.03 (д, С-9, С-33), 149.77 (д, С-10, C-32), 151.78 (с, C-16, C-26), 166.52 (c, C-8, C-34), 175.56 (c, C-19, C-23).

Общзая методика получения макроциклов 7-9. К 0.28 г (1.0 ммоль) дикетоэфира 3 в 30 мл абс. диоксана при интенсивном перемешивании медленно прибавляли 1.0 ммоль дигидразида соответствующей дикарбоновой кислоты (13-15), полученного согласно ${ }^{[13]}$. Перемешивали в течение 48 ч (контроль ТСХ), диоксан упаривали при пониженном давлении. Остаток растворяли в 20 мл $\mathrm{CH}_{2} \mathrm{Cl}_{2}$, промывали водой $(3 \times 5$ мл), сушили $\mathrm{MgSO}_{4}$ и упаривали. К полученному остатку при перемешивании добавляли последовательно 1 мл сухого $\mathrm{CH}_{2} \mathrm{Cl}_{2}$ и 10 мл гексана, выдерживали до разделения слоев, верхний из которых декантировали. Остаток промывали 5 мл гексана и упаривали в вакууме.

8,17-Диметил-1-окса-9,10,15,16-тетрааза-8,16циклотрикозадиен-2,11,14-трион (7) получен с выходом 46\%, согласно [9]. Масс-спектр (ЭУ) : $\mathrm{M}^{+} 394.2569$ (1.3, $\left.\mathrm{C}_{20} \mathrm{H}_{34} \mathrm{~N}_{4} \mathrm{O}_{4}, 394.2575\right)$, [M-C $\left.\mathrm{H}_{7} \mathrm{~N}_{2}\right]^{+} 323.1976$ (27.5, $\mathrm{C}_{17} \mathrm{H}_{27} \mathrm{~N}_{2} \mathrm{O}_{4}$,

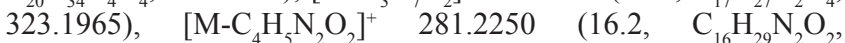

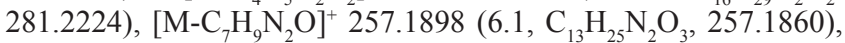
$\left[\mathrm{M}-\mathrm{C}_{8} \mathrm{H}_{15} \mathrm{~N}_{2} \mathrm{O}_{2}\right]^{+} 223.1436\left(7.9, \mathrm{C}_{12} \mathrm{H}_{19} \mathrm{~N}_{2} \mathrm{O}_{2}, 223.1441\right), 181.1689$ (2.2, $\left.\mathrm{C}_{11} \mathrm{H}_{21} \mathrm{~N}_{2}, 181.1699\right), 181.0966\left(1.7, \mathrm{C}_{9} \mathrm{H}_{13} \mathrm{~N}_{2} \mathrm{O}_{2}, 181.0972\right)$, $167.0830\left(14.1, \mathrm{C}_{8} \mathrm{H}_{11} \mathrm{~N}_{2} \mathrm{O}_{2}, 167.0815\right), 154.0733\left(52.3, \mathrm{C}_{7} \mathrm{H}_{10} \mathrm{~N}_{2} \mathrm{O}_{2}\right.$, 154.0737), 141.1400, $141.0940\left(8.6, \mathrm{C}_{8} \mathrm{H}_{17} \mathrm{~N}_{2}, 141.1386, \mathrm{C}_{8} \mathrm{H}_{13} \mathrm{O}_{2}\right.$, 141.0910), 114.0439 (39.7, $\left.\mathrm{C}_{4} \mathrm{H}_{6} \mathrm{~N}_{2} \mathrm{O}_{2}, 114.0424\right), 113$ (26.6, $\left.\mathrm{C}_{6} \mathrm{H}_{13} \mathrm{~N}_{2}, 113.1073\right), 113.0903\left(2.0, \mathrm{C}_{7} \mathrm{H}_{13} \mathrm{O}_{2}, 113.0961\right), 86.0497$ (26.0, $\left.\mathrm{C}_{3} \mathrm{H}_{6} \mathrm{NO}_{2}, 86.0475\right), 86.0337\left(2.1, \mathrm{C}_{4} \mathrm{H}_{6} \mathrm{O}_{2}, 86.0362\right), 72.0665$ $\left(100, \mathrm{C}_{3} \mathrm{H}_{8} \mathrm{~N}_{2}, 72.0682\right)$. Масс-спектры (АРCI, 20 эВ): $\mathrm{MH}^{+} 395$,

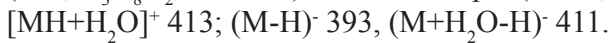

8,22-Диметил-1-окса-9, 10,20,21-тетрааза-8,21ииклооктакозадиен-2,11,19-трион (8). Выход 0.23 г (52\%). ${ }^{1} \mathrm{H}$ ЯМР $\left(\mathrm{CDCl}_{3}\right) \delta$ м.д.: 1.20-1.38 (8H, м, Н-13, Н-17, Н-26, Н-28), 1.35-1.68 (4H, м, H-5, Н-6), 1.40-1.68 (8H, м, H-4, H-14, H-15, H-16), 1.46-1.63 (4H, м, H-24, H-25), 1.73 (6H, с, $\left.\mathrm{CH}_{3}-8, \mathrm{CH}_{3}-22\right)$, 2.19 (4H, т $J=6.6$ Гц, Н-7, H-23), 2.33 (2H, т $J=6.2$ Гц, Н-3), 2.54 (4H, т $J=6.5$ Гц, H-12, H-18), 3.39 (2Н, т $J=6.5$ Гц, Н-28), 7.50 $(2 \mathrm{H}$, уш.с, $\mathrm{NH}) .{ }^{13} \mathrm{C}$ ЯMP $\left(\mathrm{CDCl}_{3}\right) \delta$ м.д.:14.77 (к, $\mathrm{CH}_{3}-8, \mathrm{CH}_{3}-$ 22), 24.11 (т, С-13, C-17), 25.40 (т, С-24), 25.61 (т, С-26), 26.36 (т, C-15), 27.96 (т, C-6), 27.97 (т, C-14, C-16), 28.13 (т, C-25), 29.19 (т, C-5), 32.05 (т, C-4), 33.53 (т, C-27), 38.13 (т, C-12, C-18), 38.45 (т, C-3), 42.17 (т, C-23), 42.86 (т, С-7), 63.59 (т, C-28), 151.35 (с, C-8, С-22), 172.20 (с, C-2), 175.71 (с, C-11, С-19). Масс-спектр (ЭУ): $\mathrm{M}^{+} 464.3376\left(53.2, \mathrm{C}_{25} \mathrm{H}_{44} \mathrm{~N}_{4} \mathrm{O}_{4}, 464.3363\right),\left[\mathrm{M}-\mathrm{CH}_{3}\right]^{+} 449.3143$ (26.2, $\left.\mathrm{C}_{24} \mathrm{H}_{41} \mathrm{~N}_{4} \mathrm{O}_{4}, 449.3128\right), 421$ (14.0), $406(9.5),\left[\mathrm{M}-\mathrm{C}_{3} \mathrm{H}_{7} \mathrm{~N}_{2}\right]^{+}$ $393.2791\left(10.8, \mathrm{C}_{22} \mathrm{H}_{37} \mathrm{~N}_{2} \mathrm{O}_{4}, 393.2753\right),\left[\mathrm{M}-\mathrm{C}_{5} \mathrm{H}_{9} \mathrm{~N}_{2} \mathrm{O}\right]^{+} 351.2654$ (7.9, $\left.\mathrm{C}_{20} \mathrm{H}_{35} \mathrm{~N}_{2} \mathrm{O}_{3}, 351.2648\right), \quad\left[\mathrm{M}-\mathrm{C}_{7} \mathrm{H}_{13} \mathrm{~N}_{2} \mathrm{O}\right]^{+} 323.2359 \quad$ (41.8, $\left.\mathrm{C}_{18} \mathrm{H}_{31} \mathrm{~N}_{2} \mathrm{O}_{3}, 323.2335\right),\left[\mathrm{M}-\mathrm{C}_{9} \mathrm{H}_{13} \mathrm{~N}_{2} \mathrm{O}_{2}\right]^{+} 283.2364\left(1.8, \mathrm{C}_{16} \mathrm{H}_{31} \mathrm{~N}_{2} \mathrm{O}_{2}\right.$,

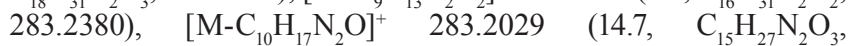

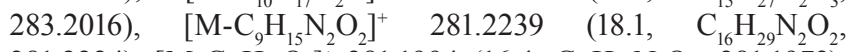
281.2224), [M- $\left.\mathrm{C}_{11} \mathrm{H}_{19} \mathrm{O}_{2}\right]^{+} 281.1994\left(16.4, \mathrm{C}_{14} \mathrm{H}_{25} \mathrm{~N}_{4} \mathrm{O}_{2}, 281.1972\right)$,

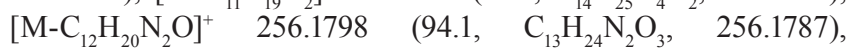
$\left[\mathrm{M}-\mathrm{C}_{13} \mathrm{H}_{23} \mathrm{~N}_{2} \mathrm{O}\right]^{+} 241.1561\left(87.2, \mathrm{C}_{12} \mathrm{H}_{21} \mathrm{~N}_{2} \mathrm{O}_{3}, 241.1552\right), 227$ (36.0), $209.1295^{23}\left(51.1, \mathrm{C}_{11} \mathrm{H}_{17} \mathrm{~N}_{2} \mathrm{O}_{2}, 209.1290\right), 185$ (20.0), 183 (21.1), 155.1197 (23.9, $\left.\mathrm{C}_{8} \mathrm{H}_{15} \mathrm{~N}_{2} \mathrm{O}, 155.1184\right), 141.0939$ (35.0, $\mathrm{C}_{8} \mathrm{H}_{13} \mathrm{O}_{2}$, 141.0916), 127 (31.2), 114.0858 (36.7, $\left.\mathrm{C}_{5} \mathrm{H}_{10} \mathrm{~N}_{2} \mathrm{O}, 114.0793\right), 109$ (20.4), 101 (19.8), $95.0689\left(50.0, \mathrm{C}_{6} \mathrm{H}_{9} \mathrm{~N}, 95.0730\right), 72$ (100), 69 (59.2), 55 (95.4). Масс-спектры (АРCI, 20 эВ): $\mathrm{MH}^{+} 465,\left[\mathrm{MH}+\mathrm{H}_{2} \mathrm{O}\right]^{+}$

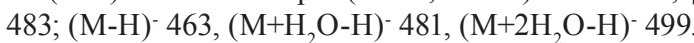

8,23-Диметил-1-окса-9, 10,20,22-тетрааза-8,22ииклононакозадиен-2,11,20-трион (9). Выход 0.26 г (57\%). ${ }^{1} \mathrm{H}$
ЯМР $\left(\mathrm{CDCl}_{3}\right)$ ठ м.д.: 1.25-1.30 (4H, м, Н-27, Н-28), 1.25-1.48 (4H, м, H-5, H-6), 1.28-1.40 (4H, м, H-13, H-18), 1.35-1.40 (2H, м, H-4), 1.48-1.70 (12H, м, H-14, H-15, H-16, H-17, H-25, H-26), 1.78 (6H, c, $\left.\mathrm{CH}_{3}-8, \mathrm{CH}_{3}-23\right), 2.29$ (2H, т $J=6.2$ Гц, H-3), 2.58 (4H, т $J=6.4$ Гц, H-12, H-19), 2.56 (4H, т $J=7.7$ Гц, Н-7, H-24), 4.20 (2Н, т $J=6.8$ Гц, Н-29), 7.60 (2Н, уш.с, NH). ${ }^{13} \mathrm{C}$ ЯMP $\left(\mathrm{CDCl}_{3}\right) \delta$ м.д.: 14.74 (к, $\left.\mathrm{CH}_{3}-8, \mathrm{CH}_{3}-23\right), 24.46$ (т, C-13, C-18), 24.71 (т, C-25), 25.01 (т, C-4), 25.47 (т, C-27), 28.07 (т, С-6, C-15, C-16), 29.20 (т, C-5), 29.25 (т, C-14, C-17), 33.59 (т, C-28), 38.09 (т, C-3), 38.21 (т, C-12, C-19), 42.92 (т, С-7, C-24), 63.68 (т, C-29), 151.22 (c, C-8, C-23), 172.99 (c, C-2), 175.70 (с, С-11, С-20). Масс-спектр (ЭУ): $\mathrm{M}^{+} 478.3525$ (64.4, $\left.\mathrm{C}_{26} \mathrm{H}_{46} \mathrm{~N}_{4} \mathrm{O}_{4}, 478.3514\right),\left[\mathrm{M}-\mathrm{CH}_{3}\right]^{+} 463.3247$ (34.9, $\mathrm{C}_{25} \mathrm{H}_{43} \mathrm{~N}_{4} \mathrm{O}_{4}$, 463.3279), [M- $\left[\mathrm{C}_{3} \mathrm{H}_{7}\right]^{+} 435.2941 \quad\left(23.7, \mathrm{C}_{23} \mathrm{H}_{39} \mathrm{~N}_{4} \mathrm{O}_{4}, 435.2966\right)$, $\left[\mathrm{M}-\mathrm{C}_{2} \mathrm{H}_{4} \mathrm{NO}\right]^{+} 420.3204\left(14.3, \mathrm{C}_{24} \mathrm{H}_{42} \mathrm{~N}_{3} \mathrm{O}_{3}, 420.3221\right),\left[\mathrm{M}-\mathrm{C}_{3} \mathrm{H}_{7} \mathrm{~N}_{2}\right]$ 407.2887 (11.5, $\left.\mathrm{C}_{23} \mathrm{H}_{39} \mathrm{~N}_{2} \mathrm{O}_{4}, 407.2904\right),\left[\mathrm{M}^{-} \mathrm{C}_{5} \mathrm{H}_{9} \mathrm{~N}_{2} \mathrm{O}\right]^{+} 365.2820$ (7.0, $\left.\mathrm{C}_{21} \mathrm{H}_{37} \mathrm{~N}_{2} \mathrm{O}_{3}, 365.2799\right)$, [M- $\left.\mathrm{C}_{7} \mathrm{H}_{11} \mathrm{~N}_{2} \mathrm{O}\right]^{+} 339.2641$ (11.2, $\mathrm{C}_{19} \mathrm{H}_{35} \mathrm{~N}_{2} \mathrm{O}_{3}$, 339.2642), [M- $\left.\mathrm{C}_{8} \mathrm{H}_{15} \mathrm{~N}_{2} \mathrm{O}\right]^{+} 323.2349\left(20.0, \mathrm{C}_{18} \mathrm{H}_{31} \mathrm{~N}_{2} \mathrm{O}_{3}, 323.2329\right)$, $\left[\mathrm{M}-\mathrm{C}_{11} \mathrm{H}_{19} \mathrm{O}_{2}\right]^{+} 295.2090\left(24.3, \mathrm{C}_{15} \mathrm{H}_{27} \mathrm{~N}_{4} \mathrm{O}_{2}, 295.2129\right), 283$ (19.8), 281 (19.1), [M- $\left.\mathrm{C}_{12} \mathrm{H}_{20} \mathrm{~N}_{2} \mathrm{O}\right]^{+} 270.1936\left(62.6, \mathrm{C}_{14} \mathrm{H}_{26} \mathrm{~N}_{2} \mathrm{O}_{3}, 270.1938\right)$, 256 (25.9), $\left[\mathrm{M}-\mathrm{C}_{13} \mathrm{H}_{23} \mathrm{~N}_{2} \mathrm{O}\right]^{+} 255.1695\left(77.7, \mathrm{C}_{13} \mathrm{H}_{23} \mathrm{~N}_{2} \mathrm{O}_{3}, 255.1703\right)$, 227 (35.3), [M- $\left.\mathrm{C}_{13} \mathrm{H}_{23} \mathrm{~N}_{2} \mathrm{O}_{3}\right]^{+} 223.1772\left(4.3, \mathrm{C}_{13} \mathrm{H}_{23} \mathrm{~N}_{2} \mathrm{O}, 223.1805\right)$, $\left[\mathrm{M}-\mathrm{C}_{14} \mathrm{H}_{27} \mathrm{~N}_{2} \mathrm{O}_{2}\right]^{+} 223.1436\left(59.8, \mathrm{C}_{12} \mathrm{H}_{19} \mathrm{~N}_{2} \mathrm{O}_{2}, 223.1441\right), 199.1314$ (26.0, $\left.\mathrm{C}_{9} \mathrm{H}_{17} \mathrm{~N}_{3} \mathrm{O}_{2}, 199.1315\right), 197.1629\left(20.5, \mathrm{C}_{11} \mathrm{H}_{21} \mathrm{~N}_{2} \mathrm{O}, 197.1648\right)$, 197.1286 (2.5, $\left.\mathrm{C}_{9} \mathrm{H}_{17} \mathrm{~N}_{2} \mathrm{O}_{2}, 197.1285\right), 167$ (24.0), 155 (25.0), 141.1401 (11.5, $\left.\mathrm{C}_{8} \mathrm{H}_{17} \mathrm{~N}_{2}, 141.1386\right), 141.0924$ (45.6, $\left.\mathrm{C}_{8} \mathrm{H}_{13} \mathrm{O}_{2}, 141.0910\right)$, 129.0922 (52.3, $\left.\mathrm{C}_{7} \mathrm{H}_{13} \mathrm{O}_{2}, 129.0910\right), 127$ (43.6), 114.0422 (40.7, $\left.\mathrm{C}_{4} \mathrm{H}_{6} \mathrm{~N}_{2} \mathrm{O}_{2}, 114.0424\right), 101$ (40.6), 95.0831 (54.8, $\left.\mathrm{C}_{7} \mathrm{H}_{11}, 95.0855\right)$, 95.0581 (13.2, $\left.\mathrm{C}_{5} \mathrm{H}_{7} \mathrm{~N}_{2}, 95.0604\right), 95.0468$ (2.9, $\left.\mathrm{C}_{6} \mathrm{H}_{7} \mathrm{O}, 95.0491\right), 85$ (25.2), 81 (27.5), 72 (88.0), 69 (100), 58 (39.6). Масс-спектры (АРСІ,

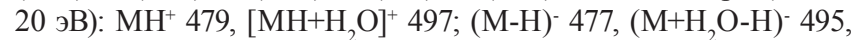
$\left(\mathrm{M}+2 \mathrm{H}_{2} \mathrm{O}-\mathrm{H}\right)^{-} 513,\left(\mathrm{M}+3 \mathrm{H}_{2} \mathrm{O}-\mathrm{H}\right)^{-} 531$.

7-Оксабицикло[2.2.1]гепт-5-ен-2,3-дигидразид

(16), 2,3,4a,5,8,8а-гексагидро-5,8-эпоксифталазин-1,4-дион (18). К 2.88 г (56.6 ммоль) 98\% $\mathrm{N}_{2} \mathrm{H}_{2} \cdot \mathrm{H}_{2} \mathrm{O}$ при интенсивном перемешивании медленно добавляли 3.00 г (14.2 ммоль) диметилового эфира 7-оксабицикло[2.2.1]гепт-5-ен-2,3-дикарбоновой кислоты (17). Полученный белый осадок отфильтровывали, промывали $\mathrm{Et}_{2} \mathrm{O}$, получили 1.26 г (42\%) соединения 16. Т. пл. $142.5-143.5^{\circ} \mathrm{C}$. ИК (KBr) $v_{\max } \mathrm{cm}^{-1}: 3282(\mathrm{NH}), 1649(\mathrm{CONH})$. ${ }^{1} \mathrm{H}$ ЯМР (D,O) $\delta$ м.д.: 3.12 (2H, c, H-2, H-3), 4.93 (2H, c, H-1, $\mathrm{H}-4), 5.27$ (6H, уш.с, $\left.\mathrm{NHNH}_{2}\right), 6.91(2 \mathrm{H}, \mathrm{c}, \mathrm{H}-5, \mathrm{H}-6) .{ }^{13} \mathrm{C}$ ЯМР $\left(\mathrm{D}_{2} \mathrm{O}\right): 46.78$ (C-2, C-3), 82.06 (C-1, C-4), 134.09 (C-5, C-6), 178.70 (CONH). Масс-спектр (АРCI, 20 эВ): 213 [(М+H $\left.{ }^{+}\right], 231$ $\left[\left(\mathrm{M}+\mathrm{H}^{+} \mathrm{H}_{2} \mathrm{O}\right)^{+}\right], 249\left[\left(\mathrm{M}+\mathrm{H}+2 \mathrm{H}_{2} \mathrm{O}\right)^{+}\right]$.

Фильтрат упаривали и получили 1.37 г (54\%) соединения 18. Т. пл. $120.5-122.5^{\circ} \mathrm{C}$. ИК (KBr) $v_{\max } \mathrm{cm}^{-1}: 3196(\mathrm{NH}), 1687$ (CONH). ${ }^{1} \mathrm{H}$ ЯМР $\left(\mathrm{D}_{2} \mathrm{O}\right) \delta$ м.д.: $3.03(2 \mathrm{H}, \mathrm{c}, \mathrm{H}-4 \mathrm{a}, \mathrm{H}-8 \mathrm{a}), 5.27$ (2H, c, H-5, H-8), 6.58 (2H, c, H-6, H-7), 8.00-9.80 (2H, уш.c, $\mathrm{NH}) .{ }^{13} \mathrm{C}$ ЯMP (D 20$): 49.54$ (C-4a, C-8a), 80.06 (C-5, C-8), 137.59 (C-6, С-7), 165.74 (С-1, С-4). Масс-спектр (АРСІ, 20 эВ): 181 $\left[(\mathrm{M}+\mathrm{H})^{+}\right], 199\left[\left(\mathrm{M}+\mathrm{H}+\mathrm{H}_{2} \mathrm{O}\right)^{+}\right], 179\left[(\mathrm{M}-\mathrm{H})^{-}\right]$.

4,25-Диметил-28а,29,32,32а-тетрагидро-29,32эпокси-11,18,2,3,26,27-бензадиоксатетраазаииклотриаконт a-3,25-диен-1,12,17,28-тетраон (11). К 0.80 г (2.00 ммоль) дикетодиэфира 4 в 13 мл абс. диоксана при интенсивном перемешивании медленно прибавляли 0.42 г (2.0 ммоль) дигидразида 7-оксабицикло[2.2.1]гепт-5-ен-2,3-дикарбоновой кислоты (16). Перемешивали в течение 48 ч (контроль ТCX), диоксан упаривали. Остаток растворяли в 20 мл $\mathrm{CH}_{2} \mathrm{Cl}_{2}$, промывали водой $(3 \mathrm{x}$ 3 мл), сушили $\mathrm{MgSO}_{4}$ и упаривали. К полученному остатку при перемешивании добавляли последовательно 1 мл абс. $\mathrm{CH}_{2} \mathrm{Cl}_{2}$ и 10 мл гексана, выдерживали до разделения слоев, верхний из которых декантировали. Остаток промывали 3 мл гексана и упаривали. Выход 0.35 г (64\%). ИК (KBr) $v_{\max } \mathrm{cm}^{-1}: 3329(\mathrm{NH})$, $1730(\mathrm{O}=\mathrm{C}-\mathrm{O}), 1699(\mathrm{CONH}), 1647(\mathrm{C}=\mathrm{C}), 1635(\mathrm{C}=\mathrm{N}) .{ }^{1} \mathrm{H}$ ЯMP $\left(\mathrm{CDCl}_{3}\right) \delta$ м.д.: $1.30-1.60$ (8H, м, H-6, H-9, H-20, H-23), 1.60-1.75 (4H, м, H-14, Н-15), 1.61-1.80 (8H, м, Н-7, Н-8, Н-21, Н-22), 
$2.10\left(6 \mathrm{H}, \mathrm{c}, \mathrm{CH}_{3}\right), 2.37$ (4H, т $J=6.7$ Гц, Н-13, H-16), 2.52 (4H, т $J=7.5$ Гц, Н-5, H-24), 4.12 (4Н, т $J=6.9$ Гц, Н-10, Н-19), 4.31 (2H, д $J=4.0$ Гц, Н-28a, H-32a), 5.34 (2H, д $J=4.0$ Гц, Н-29, Н-32), $6.60(2 \mathrm{H}, \mathrm{c}, \mathrm{H}-30, \mathrm{H}-31), 8.10(2 \mathrm{H}$, уш.c, $\mathrm{NH}) .{ }^{13} \mathrm{C}$ ЯMP $\left(\mathrm{CDCl}_{3}\right.$ (DMSO-d $)$ ): 16.32 (16.48) $\left(\mathrm{CH}_{3}\right), 22.52(22.15)\left(\mathrm{CH}_{3}\right), 24.08(\mathrm{C}-7$, C-8, C-21, C-22), 25.41 (C-6, C-23), 28.58 (C-14, C-15), 33.57 (C-9, C-20), 38.22 (C-13, C-16), 39.49 (C-5, C-24), 48.68 (45.87) (C-28a, C-32a), 64.04 (C-10, C-19), 80.46 (78.64) (C-29, C-32), 135.98 (C-30, C-31), 162.41 (162.41) (C-4, C-25), 171.08 (171.84) (C-1, C-28), 173.05 (C-12, C-17). Масс-спектр (АРСI, 20 эВ): $575\left[(\mathrm{M}+\mathrm{H})^{+}\right], 593\left[\left(\mathrm{M}+\mathrm{H}+\mathrm{H}_{2} \mathrm{O}\right)^{+}\right], 573\left[(\mathrm{M}-\mathrm{H})^{-}\right]$.

\section{Список литературы}

\section{References}

1. Ishmuratov G.Yu., Yakovleva M.P., Tambovtsev K.A., Legostaeva Yu.V., Kravchenko L.V., Ishmuratova N.M., Tolstikov G.A. Chem. Nat. Compd. 2008, 44, 74-76.

2. Ishmuratov G.Yu., Yakovleva M.P., Gareeva G.R., Kravchenko L.V., Ishmuratova N.M., Talipov R.F. Bashkir University Bulletin 2008, 3, 466-469 (in Russ.).

3. Ishmuratov G.Yu., Mingaleeva G.R., Yakovleva M.P., Muslukhov R.R., Kashipov R.N., Ishmuratova N.M., Tolstikov A.G. Butlerov Communications 2009, 17(5), 35-38 (in Russ.).

4. Ishmuratov G.Yu., Ismagilova A.F., Mingaleeva G.R., Chudov I.V., Yakovleva M.P., Muslukhov R.R., Kashipov R.N.,
Tolstikov A.G. Butlerov Communications 2009, 16(4), 21-25 (in Russ.).

5. Sprague P.W., Heikes J.E., Gougoutas J.Z., Malley M.F., Harris D.N., Greenberg R. J. Med. Chem. 1985, 28, 1580-1590.

6. Suzuki T., Kagaya S., Tomino A., Unno K., Kametani T., Takahashi T., Tanaka Y. Heterocycles 1978, 9, 1749-1757.

7. Sprague P.W., Heikes J.E., Harris D.N., Greenberg R. Adv. Prostaglandiun and Thromboxane Res. 4th Int. Prostahlandin Conf., Washington, D. C. 1979, Vol. 6.; New York. 1980. p. 493-496.

8. Obshchaya Organicheskaya Khimiya, Moskva: Khimiya, 1983, Vol 4. 438 p. Russian translation of Comprehensive Organic Chemistry: The Synthesis and Reactions of Organic Compounds [Barton D., Ollis W.D., Eds.] Vol. 4: Heterocyclic Compounds. Oxford: Pergamon Press, 1979.

9. Ishmuratov G.Yu., Yakovleva M.P., Mingaleeva G.R., Muslukhov R.R., Vyrypaev E.M., Galkin E.G., Ivanov S.P., Tolstikov A.G. Chem. Nat. Compd. 2009, 45, 465-469.

10. Traldi P., Vettori U., Podda G., Maccioni A., Corda L. Org. Mass Spectrom. 1983, 18(2), 69-74.

11. Takhistov V.V., Ponomarev D.A. Organicgeskaya Massspektrometriya [Organic Mass-spectrometry] Sankt-Peterburg: VVM, 2005. 344 p. (in Russ.).

12. Timofeev O.S. Mass-spectra and Ion-molecular Reactions of Macroheterocycles in Gas Phase. Diss. Can. Chem. Sci. Odessa, 1985 (in Russ.).

13. Weygand-Hilgetag. Organisch-Chemische Experimentierkunst. Leipzig: Verlag, 1964. . 